\title{
Triangular-Hamate Joint
}

National Cancer Institute

\section{Source}

National Cancer Institute. Triangular-Hamate Joint. NCI Thesaurus. Code C139200.

The articulation between the hamate and the triquetral bones in the wrist. 